\title{
The Wetting Process of a Dry Polymeric Hydrogel
}

\author{
Shichen JI and Jiandong DING ${ }^{\dagger}$ \\ Department of Macromolecular Science, Key Laboratory of Molecular Engineering of Polymers, Fudan University, \\ Shanghai 200433, China
}

(Received November 12, 2001; Accepted March 5, 2002)

\begin{abstract}
The swelling of a dry cylindrical poly(acrylic acid-co-acrylamide) gel was observed in real time after immersed in water. Pattern formation was found during this process. The wetting process is divided into three stages: first, swelling in the radial direction with cusp-like patterns evolving on the surface; second, shrinking in the radial direction while swelling in the axial direction; the last one, re-swelling in the radial direction. The movement of the wetting front at the first stage exhibits Case II like diffusion of water in polymeric network.
\end{abstract}

KEY WORDS Hydrogel / Wetting / Swelling / Pattern Formation / Case II Diffusion /

Polymer gel is a three dimensional network filled with solvent. In many cases, hydrogel undergoes extensive volume change in response to external stimuli. ${ }^{1-14}$ Various kinds of patterns have also been observed if the volume changes fast enough in either swelling or shrinking. ${ }^{2-9}$ The pattern formation has been intensively studied since it is believed helpful to elucidate the mechanical properties of gels and pattern evolutions in biological systems. ${ }^{2-7}$

Our previous paper reported a novel pattern, gel helix, formed during a rapid shrinking of poly(acrylic acid-co-acrylamide) (P(AA-co-Am)) gel cylinders ${ }^{8}$ together with bamboo like pattern. ${ }^{9}$ This paper touches on a more fundamental and "simple" process of hydrogels, wetting process of a dry hydrophilic polymer gel immersed in water. Complicated patterns, though different from gel helix, are observed together with the movement of a wetting front which exhibits kinetic behaviors of Case II like diffusion.

\section{EXPERIMENTAL}

The gel was synthesized by a traditional method as reported previously. ${ }^{8}$ Purified acrylic acid $(1.53 \mathrm{~g})$, acrylamide $(1.52 \mathrm{~g})$ and purified $N, N^{\prime}$-(methylene bisacrylamide) $(83.3 \mathrm{mg})$ were dissolved in $10 \mathrm{~mL}$ distilled water. After adding $25.7 \mathrm{mg}$ of purified ammonium persulfate, glass capillaries $(0.2-0.5 \mathrm{~mm})$ were dipped into the solution. The polymerization was carried out for $1 \mathrm{~h}$ at $40^{\circ} \mathrm{C}$ followed by $4 \mathrm{~h}$ at $60^{\circ} \mathrm{C}$.

The P(AA-co-Am) gel cylinders were obtained by gently removed from the capillaries. To enhance contrast of the transparent entity, the gel was immersed in water, containing a small amount of dyes, methylene blue overnight. It was dried in the air at room temperature for $12 \mathrm{~h}$, and further dried $70^{\circ} \mathrm{C}$ for $10 \mathrm{~min}$. The dried gel was put into water and observed in a Leitz optical microscope equipped with a self-built CCD camera. The gel wetting process was recorded in real time by the video camera.

\section{RESULTS AND DISCUSSION}

A typical morphology of a gel cylinder during wetting process is shown in Figure 1. A sharp wetting front between an internal unswollen core and external swollen region was clearly observed. The diameter of the internal core $\left(d_{\text {int }}\right)$ and that of the cylindrical gel $\left(d_{\text {ext }}\right)$ along a certain cross section are indicated in Figure 1b. Swelling patterns of hydrogels have been reported in the literature, where the polymeric gels are usually spherical or slab-like instead of cylindrical and swelling processes are initiated from a real hydrogel containing water instead of a dry gel. ${ }^{2,3}$ A typical swelling process has been reported by Tanaka et al., ${ }^{2,3}$ when a spherical hydrogel with smooth surface swells,

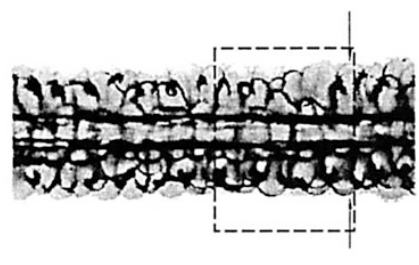

(a)

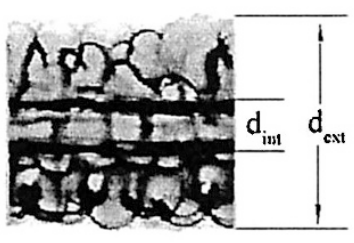

(b)
Figure 1. (a) A typical pattern during the wetting process of a dry $\mathrm{P}(\mathrm{AA}-\mathrm{co}-\mathrm{Am})$ gel cylinder with clear wetting front, and (b) magnification of the marked part in (a) demonstrating the definition of gel external diameter, $d_{\mathrm{ext}}$ and internal diameter for the unswollen part, $d_{\text {int }}$.

†'To whom correspondence should be addressed (E-mail: jdding1@fudan.edu.cn). 


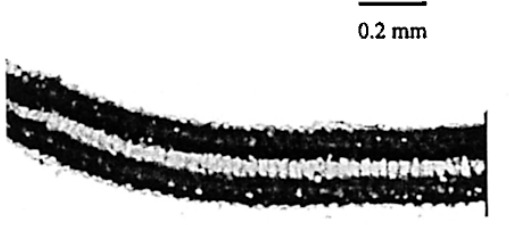

(a) $45 \mathrm{~s}$

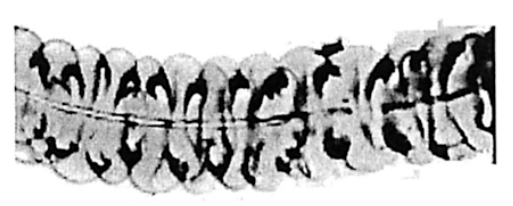

(d) $164 \mathrm{~s}$

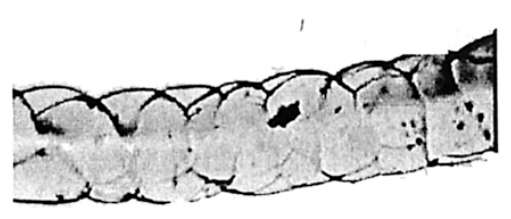

(g) $191 \mathrm{~s}$

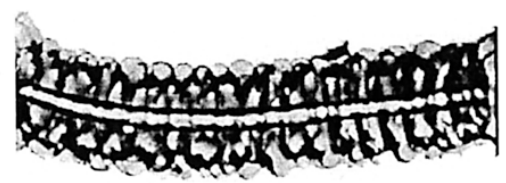

(b) $112 \mathrm{~s}$

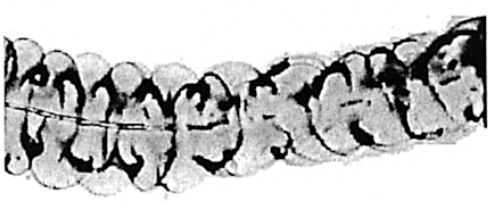

(e) $171 \mathrm{~s}$

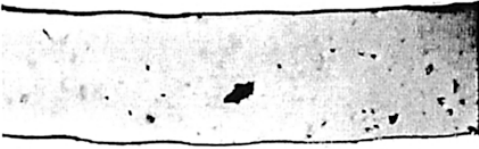

(h) $230 \mathrm{~s}$

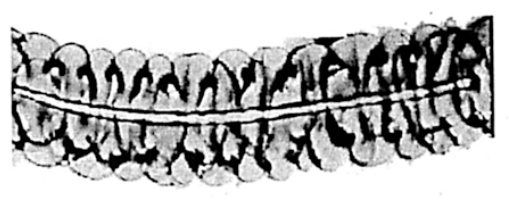

(c) $144 \mathrm{~s}$

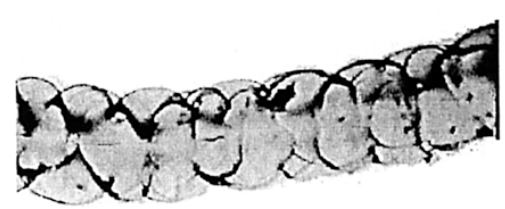

(f) $176 \mathrm{~s}$

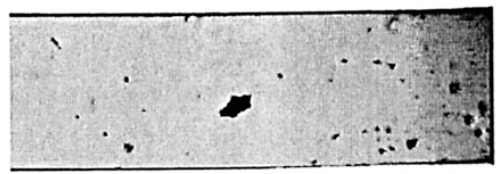

(i) $406 \mathrm{~s}$

Figure 2. Real time wetting process of a cylindrical $\mathrm{P}(\mathrm{AA}-\mathrm{co}-\mathrm{Am})$ hydrogel dried at room temperature for $12 \mathrm{~h}$ and then $70^{\circ} \mathrm{C}$ for $10 \mathrm{~min}$.

regular patterns was observed on the surface, then coalesced and eventually disappeared. Permanent patterns are also observed on mechanically constrained gel slab. ${ }^{3}$ In our experiment, the unswollen core constrains the swelling region and thus leads to pattern formation in the wetted region. Therefore, the pattern formation in our gel cylinders is essentially similar to those in gel slabs and gel spheres.

The gel cylinder swells with fine patterns appearing on the surface after dipped in water (Figure 2a). The contour of the gel cylinder is sinusoidal, similar to the simulated result of a two dimensional elastic network with the upper surface free and the lower surface constrained. ${ }^{10}$ Fine cusp-like patterns become larger by coalescing with each other as time goes on (Figure $2 b-c$ ).

Through the wetting experiments of dry cylindrical gels, we found some interesting phenomena besides pattern formation of gel in swelling, namely, movement of a sharp wetting front and gel shrinking during the global swelling. At the first stage of gel wetting, a continuous decrease of $d_{\text {int }}$ and increase of $d_{\text {ext }} v s$. time

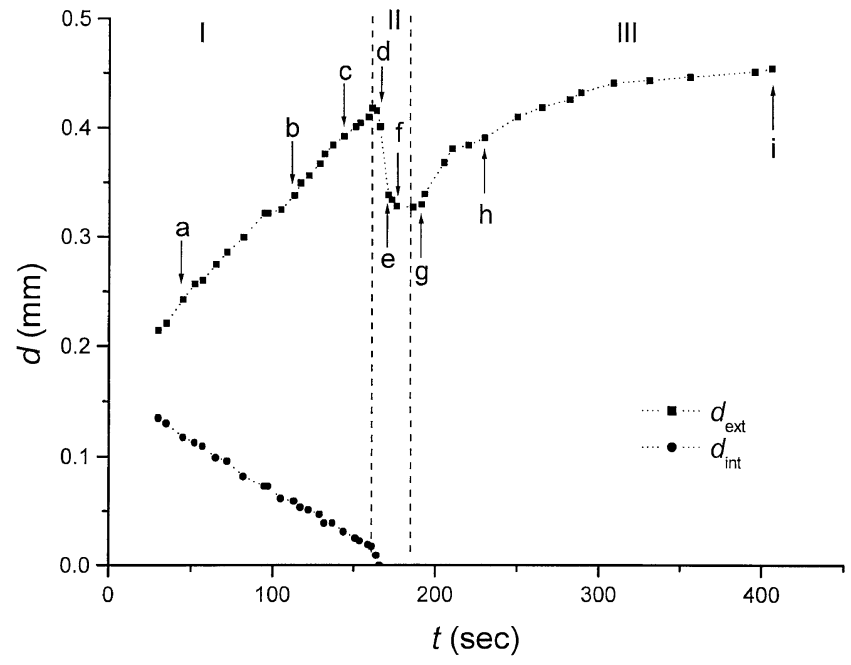

Figure 3. Time evolution of $d_{\mathrm{ext}}$ and $d_{\text {int }}$ in the wetting process of the gel cylinder with the associated morphologies shown in Figure 2 .

was observed, which indicates that the wetting front advances towards the center at a constant velocity (Figure 3$)$. When the $d_{\text {int }}$ is near zero, the core is broken; 
the gel shrinks in the radial direction (Figure $2 \mathrm{~d}$ to $2 \mathrm{e}$ and Figure 3). We thus describe this phenomenon as "a shrinking intermediate during swelling process". After that, the gel swells again. The complicated patterns disappear eventually with the final homogenous equilibrium state (Figure $2 \mathrm{f}$ to $2 \mathrm{i}$ ). According to the changes of $d_{\text {int }}$ and $d_{\text {ext }}$, we classify the wetting process of a dry polymeric hydrogel cylinder into three stages: first, swelling in the radial direction with cusp-like patterns evolving on the surface; second, shrinking in the radial direction while swelling in the axial direction; the last one, re-swelling in the radial direction. The first stage is associated with the movement of the wetting front together with pattern formation. The second stage is initiated after wetting fronts from different radial directions meet with each other. Due to the disappearance of the constraint to the wetting and swelling region, the gel is elongated along the axial direction. Such an abrupt elongation leads to shrinkage along the radial direction. The last stage is quite usual and just results from a "normal" swelling of a hydrogel.

The phenomenon of a reduction intermediate in film thickness has been reported in the swelling process of a PMMA sheet in an organic solvent, methanol, when Thomas and Windle studied the Case II diffusion of glassy polymers. ${ }^{15,16}$ But no patterns have been observed in their solvent-penetration process because PMMA swells very slowly (tens of hours) in methanol. According to the diffusion theory, ${ }^{17}$ solvent penetration in a polymer network can be classified into three cases: Fickian (Case I) diffusion, non-Fickian (Case II) diffusion and anomalous diffusion, originally proposed by Alfrey et al. ${ }^{18}$ Such a classification can be judged by $M_{\mathrm{t}}$, the amount of solvent absorbed per unit area of polymer related to $t$, the diffusion time as

$$
M_{\mathrm{t}}=k t^{n}
$$

where $k$ is a constant and the exponent $n$ underlies the diffusion mechanism. Case I and Case II diffusions result in $n=0.5$ and 1 , whereas the anomalous diffusion refers to $0.5<n<1$. The existence of a sharp wetting front and its linear movement with time are two most characteristic phenomenological features of Case II diffusion, which does be the case of the first-stage wetting of our dry gel cylinder (Figures 2 and 3).

Non-Fickian diffusion occurs mainly in glassy polymers, when the temperature of study is much below the glassy transition temperature $\left(T_{\mathrm{g}}\right)$. In our experiment, the dried P(AA-co-Am) gel is in the glassy state, because its glass transition temperature, which is between that of Poly(acrylic acid) $(379 \mathrm{~K})$ and that of Poly(acrylamide) $(438 \mathrm{~K}),{ }^{19}$ is well above the room temperature. For any polymer-penetrant system, the

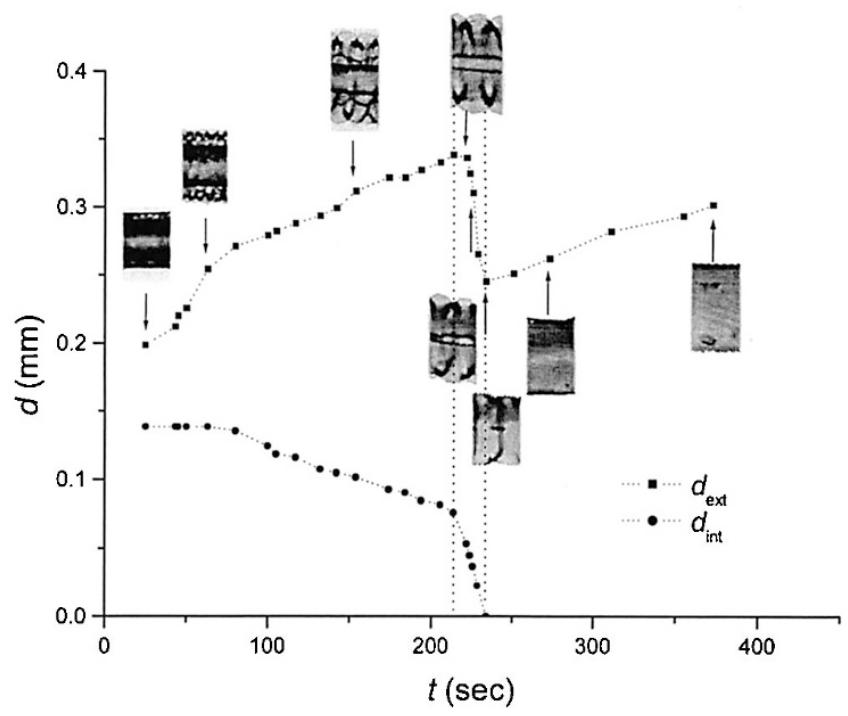

Figure 4. The diameter change of a $\mathrm{P}(\mathrm{AA}-\mathrm{co}-\mathrm{Am})$ gel cylinder dried only at room temperature for $12 \mathrm{~h}$. The morphologies of the gel are shown along with the evolution curve.

process in which the penetrant substantially swells the polymer is a combination of solvent diffusion and polymer relaxation. The essential reason for Case II diffusion is that the polymer relaxation lags much behind penetrant diffusion. In our experiment, the solvent penetration is much fast after a dry "hydrogel" is re-immersed in water. The underlying wetting process is thus a case II diffusion. In contrast to it, if the gel cylinder is not dry sufficiently, the wetting process (Figure 4) does not agree with the standard Case II diffusion completely and is only an anomalous one, although it is still somehow Case II like. A small amount of water in the dry gel may be regarded as plasticizer and thus enhances the chain relaxation or relatively depresses water penetration.

\section{CONCLUSIONS}

The wetting process of a dry cylindrical hydrogel has been observed in real time. Shrinking during swelling process has been reproduced. The wetting process of a dry gel cylinder is divided into three stages: first, gel swells in the radial direction with cusp-like patterns evolving on the surface; second, gel shrinks in the radial direction and swells in the axial direction; third, gel re-swells to the final state and patterns eventually disappear. The sharp wetting front is observed at the first stage with linear movement with time, if the gel is dry sufficient. A Case II like diffusion of water in dried "hydrogel" is thus found along the complicated pattern formation. The amount of residue water seems to affect the diffusion type. The research may help to a better understanding of "anomalous diffusion" in polymers. 
We believe that in most of cases, the wetting process of dried hydrogel might exhibit an anomalous diffusion behaviors instead of those of either a Case I diffusion or a perfect Case II diffusion.

The theory of Case II diffusion for a polymerpenetrant system without pattern formation has been set up by Thomas and Windle. ${ }^{15}$ Swelling process of a gel undergoing volume transition has also been dealt with in the literature. ${ }^{10,12,13,20}$ Nevertheless, the wetting process of a polymeric hydrogel associated with complicated pattern formation has not yet been satisfactorily treated in theory. Further extensive investigation is thus required.

Acknowledgment. This work was financially supported by NSF of China, the National Basic Science Research and Development Grants (973), 863 projects, and the Award Foundation for Young Teachers from Ministry of Education.

\section{REFERENCES}

1. Y. Osada and J. Gong, Prog. Polym. Sci., 13, 187 (1993).

2. T. Tanaka, Physica, 140A, 261 (1986).
3. T. Tanaka, S. T. Sun, Y. Hirokawa, S. Katayama, J. Kucera, Y. Hirose, and T. Amiya, Nature, 325, 796 (1987).

4. E. S. Matsuo and T. Tanaka, Nature, 358, 482 (1992).

5. Y. Li, C. Li, and Z. Hu, J. Chem. Phys., 100, 4637 (1994).

6. A. Suzuki, S. Yoshikawa, and G. Bai, J. Chem. Phys., 111, 360 (1999).

7. G. Bai and A. Suzuki, J. Chem. Phys., 111, 10338 (1999).

8. S. Ji and J. Ding, Polym. J., 33, 701 (2001).

9. S. Ji and J. Ding, Chem. J. Chinese Univ., 22, 1254 (2001) (Chinese).

10. K. Sekimoto and K. Kawasaki, J. Phys. Soc. Jpn., 57, 2594 (1988).

11. A. Onuki, J. Phys. Soc. Jpn., 57, 703 (1987).

12. T. Hwa and M. Kardar, Phys. Rev. Lett., 61, 106 (1988).

13. N. Suematsu, K. Sekimoto, and K. Kawasaki, Phys. Rev. A, 41, 5751 (1990).

14. E. S. Matsuo and T. Tanaka, J. Chem. Phys., 89, 1695 (1988).

15. N. L. Thomas and A. H. Windle, Polymer, 23, 529 (1982).

16. N. L. Thomas and A. H. Windle, Polymer, 22, 627 (1981).

17. L. Masaro, and X. X. Zhu, Prog. Polym. Sci., 24, 731 (1999).

18. T. Alfrey, E. F. Gurnee, and W. G. Lloyd, J. Polym. Sci., C, 12, 249 (1966).

19. J. Brandrup and E. H. Immergut, "Polymer Handbook, second ed.," John Wiley \& Sons, New York, N.Y., 1974, III-144, III146.

20. T. Tomari and M. Doi, J. Phys. Soc. Jpn., 63, 2093 (1994). 